\title{
ON A GENERALISATION OF A RESULT OF RAMANUJAN CONNECTED WITH THE EXPONENTIAL SERIES
}

\author{
by R. B. PARIS
}

(Received 6th June 1980)

\section{Introduction}

One of the many interesting problems discussed by Ramanujan is an approximation related to the exponential series for $e^{n}$, when $n$ assumes large positive integer values. If the number $\theta_{n}$ is defined by

$$
\frac{1}{2} e^{n}=\sum_{k=0}^{n-1} \frac{n^{k}}{k !}+\frac{n^{n}}{n !} \theta_{n}(n \geqq 1), \quad \theta_{0}=\frac{1}{2}
$$

Ramanujan (9) showed that when $n$ is large, $\theta_{n}$ possesses the asymptotic expansion

$$
\theta_{n}=\frac{1}{3}+\frac{4}{135 n}-\frac{8}{2835 n^{2}}-\frac{16}{8505 n^{3}}+\ldots
$$

The first demonstrations that $\theta_{n}$ lies between $\frac{1}{2}$ and $\frac{1}{3}$ and that $\theta_{n}$ decreases monotonically to the value $\frac{1}{3}$ as $n$ increases, were given by Szegö (12) and Watson (13). Analogous results were shown to exist for the function $e^{-n}$, for positive integer values of $n$, by Copson (4). If $\phi_{n}$ is defined by

$$
e^{-n}=\sum_{k=0}^{n-1} \frac{(-n)^{k}}{k !}+\frac{(-n)^{n}}{n !} \phi_{n}(n \geqq 1), \quad \phi_{0}=1
$$

then $\phi_{n}$ lies between 1 and $\frac{1}{2}$ and tends monotonically to the value $\frac{1}{2}$ as $n$ increases, with the asymptotic expansion

$$
\phi_{n}=\frac{1}{2}+\frac{1}{8 n}+\frac{1}{32 n^{2}}-\frac{1}{128 n^{3}}-\frac{13}{512 n^{4}}+\ldots
$$

A generalisation of these results was considered by Buckholtz (2) who defined, in a slightly different notation, for complex $z$ and positive integer $n$, the function $\phi_{n}(z)$ by

$$
e^{n z}=\sum_{k=0}^{n-1} \frac{(n z)^{k}}{k !}+\frac{(n z)^{n}}{n !} \phi_{n}(z) .
$$

As $n \rightarrow \infty$, Buckholtz showed that $\phi_{n}(z)$ possesses the asymptotic expansion

$$
\phi_{n}(z) \sim \sum_{m=0}^{\infty} c_{m}(z) n^{-m},
$$


uniformly valid for all complex values of $z$ except in the neighbourhood of the point $z=1$. The coefficients $c_{m}(z)$ are defined by

$$
c_{m}(z)=\frac{(-)^{m} P_{m}(z)}{(1-z)^{2 m+1}}
$$

where $P_{m}(z)$ is a polynomial in $z$ of degree $m$ with positive integral coefficients. For the first few values of $m$ we have

$$
\begin{array}{ll}
P_{0}(z)=1, \quad P_{1}(z)=z, & P_{2}(z)=z+2 z^{2} \\
P_{3}(z)=z+8 z^{2}+6 z^{3}, & P_{4}(z)=z+22 z^{2}+58 z^{3}+24 z^{4}
\end{array}
$$

The polynomials $P_{m}(z)$ have been discussed in further detail by Carlitz (3). When $z=-1$ the expansion (1.6) reduces to that found in (1.4) by Copson.

An asymptotic expansion of $\phi_{n}(z)$ which is uniformly valid as $n \rightarrow \infty$ has been obtained by Wong (15), for real values of $z(=x)$ restricted to the interval $\delta \leqq x \leqq 1$, where $\delta$ is some fixed positive number. The leading term of this expansion is expressed in terms of the Weber parabolic cylinder function by

$$
\phi_{n}(x) \sim 1+\frac{x}{1-x}(n \alpha)^{1 / 2} \exp \left(\frac{1}{4} n \alpha\right) D_{-1}\left((n \alpha)^{1 / 2}\right) \quad n \rightarrow \infty
$$

where $\alpha=2(x-1-\log x)$. This expansion may be shown to yield Ramanujan's result (1.2) when $x=1$ and that given by Buckholtz in (1.6) for $x<1$.

Ramanujan's result is of interest since it enables $e^{n}$ to be expressed between rational limits and finds application in certain algorithms in numerical computation (7, p. 112). It shows that the remainder function $\theta_{n}$, after truncation of the series for $e^{n}$ at its maximum term $n^{n} / n$ !, is $O(1)$ for $n \rightarrow \infty$. For large $n$ the sum of the first $n$ terms is thus roughly half the sum of the whole series. A result of this type was, in fact, employed earlier by Stokes (11) in the determination of the asymptotic behaviour of the generalised hypergeometric function (10, p. 40)

$$
{ }_{\mathrm{p}} F_{\mathrm{q}}\left(a_{1}, \ldots, a_{\mathrm{p}} ; b_{1}, \ldots, b_{q} ; x\right)=\sum_{k=0}^{\infty} \frac{\prod_{r=1}^{p}\left(a_{r}\right)_{k}}{\prod_{r=1}^{a}\left(b_{r}\right)_{k}} \frac{x^{k}}{k !} \quad(q \geqq p \geqq 0)
$$

for $x \rightarrow+\infty$ and real positive values of the parameters $a_{r}$ and $b_{r}$. It would therefore seem probable that the character exhibited by the remainder functions $\theta_{n}$ and $\phi_{n}$ for the exponential function is of a more general nature, and that results analogous to those of Ramanujan and Copson might be expected to exist for more complicated transcendental functions.

In this paper we consider, as a generalisation of the exponential function, the confluent hypergeometric functions ${ }_{1} F_{1}(a ; b ; \pm n)$ which reduce to $e^{ \pm n}$ respectively when $a=b$ and are defined by (1.9) with $p=q=1$. For convenience, the parameters $a$ and $b$ will be supposed throughout to be real, although the analysis may be extended to complex values without any complication. We show, by defining remainder functions for the truncated hypergeometric functions analogous to those in (1.1) and (1.3), that similar results for large values of $n$ also exist for this class of transcendental function. 
The problem is reduced to that of the examination of the asymptotic nature of a higher order confluent function of the type ${ }_{2} F_{2}( \pm n)$, when some of the parameters and also the argument assume large values.

A discussion of the Bessel function $J_{0}(\lambda n)$ where $\lambda$ is restricted to the range $0<\lambda \leqq 1$ is given as a further example of a generalisation of Copson's results.

\section{The hypergeometric function ${ }_{1} F_{1}(a ; b ;-n)$}

We first consider the hypergeometric function ${ }_{1} F_{1}(a ; b ;-n)$, where the parameters $a$ and $b$ are supposed to be real. We suppose further that neither of $a$ and $b$ is equal to zero or a negative integer, since when $a=-k, k=0,1,2, \ldots$ the hypergeometric function reduces to a polynomial of degree $k$ and when $b=-k$ the function is no longer defined (unless also $a=-k+m, m=0,1,2, \ldots, k$ ).

We define the remainder function $\psi_{n}$ by

$$
{ }_{1} F_{1}(a ; b ;-n)=\sum_{k=0}^{n-1} \frac{(a)_{k}}{(b)_{k}} \frac{(-n)^{k}}{k !}+\frac{(a)_{n}(-n)^{n}}{(b)_{n} n !} \psi_{n}(n \geqq 1), \psi_{0}=1
$$

where $(a)_{k}$ denotes $\Gamma(a+k) / \Gamma(a)$. It is readily shown, by use of the result $(a)_{n+m}=$ $(a)_{n}(a+n)_{m}$ for positive integral $m$ combined with the series representation of ${ }_{1} F_{1}(a ; b ;-n)$ in $(1.9)$, that

$$
\begin{aligned}
\psi_{n} & =1-\frac{a+n}{b+n} \frac{n}{n+1}+\frac{(a+n)_{2}}{(b+n)_{2}} \frac{n^{2}}{(n+1)(n+2)}-\ldots \\
& ={ }_{2} F_{2}(1, a+n ; b+n, n+1 ;-n) .
\end{aligned}
$$

We observe that when $a=b$, corresponding to Copson's case (1.3), the ${ }_{2} F_{2}$ function contracts to yield the remainder function $\psi_{n} \equiv \phi_{n}$ given by

$$
\psi_{n}={ }_{1} F_{1}(1 ; n+1 ;-n)=(-)^{n} \frac{e^{-n} n !}{n^{n}}\left\{1-\frac{\Gamma(n,-n)}{\Gamma(n)}\right\},
$$

where $\Gamma(a, z)$ denotes the incomplete gamma function, and that a similar simplification occurs when $a=1$.

Employing the Euler-type integral representation for the generalised hypergeometric function (10, p. 108)

$$
\begin{aligned}
& { }_{p+1} F_{q+1}\left(\alpha, a_{1}, \ldots, a_{p} ; \alpha+\beta, b_{1}, \ldots, b_{q} ; z\right) \\
& =\frac{\Gamma(\alpha+\beta)}{\Gamma(\alpha) \Gamma(\beta)} \int_{0}^{1} t^{\alpha-1}(1-t)^{\beta-1} F_{q}\left(a_{1}, \ldots, a_{p} ; b_{1}, \ldots, b_{q} ; z t\right) d t, \\
& \quad \operatorname{Re}(\alpha)>0, \operatorname{Re}(\beta)>0, p \leqq q+1
\end{aligned}
$$

we may express $\psi_{n}, n=1,2, \ldots$, as an integral of the form

$$
\begin{aligned}
\psi_{n} & =n \int_{0}^{1}(1-x)^{n-1} F_{1}(a+n ; b+n ;-n x) d x \\
& =\frac{n \Gamma(b+n)}{\Gamma(b-a) \Gamma(a+n)} \int_{0}^{1} \int_{0}^{1}(1-x)^{n-1} y^{a+n-1}(1-y)^{b-a-1} e^{-n x y} d x d y,
\end{aligned}
$$


the latter integral being convergent for $b>a$ and $a>-n$. Integrating (2.6) by parts to find

$$
\psi_{n}=1-\frac{n \Gamma(b+n)}{\Gamma(b-a) \Gamma(a+n)} \int_{0}^{1} \int_{0}^{1}(1-x)^{n} y^{a+n}(1-y)^{b-a-1} e^{-n x y} d x d y
$$

and adding this result to (2.6), we obtain

$$
\psi_{n}=\frac{1}{2}+\frac{1}{2} \frac{n \Gamma(b+n)}{\Gamma(b-a) \Gamma(a+n)} \int_{0}^{1} \int_{0}^{1}(1-x)^{n-1} y^{a+n-1}(1-y)^{b-a-1}[1-(1-x) y] e^{-n x y} d x d y
$$

for $b>a$ and $a>-n, n=1,2, \ldots$ Since the integrands in (2.7) and (2.8) are both non-negative over the domain of integration, and from (2.1) $\psi_{0}=1$, we deduce that $\frac{1}{2}<\psi_{n} \leqq 1$ for $n=0,1,2, \ldots$ when $b>a>-1$.

We now proceed to determine the asymptotic expansion of $\psi_{n}$ for $n \rightarrow \infty$. We reverse the order of integration in (2.6), when $b>a$ and $a>-n$, to find

$$
\psi_{n}=\frac{n}{\Gamma(b-a)} \frac{\Gamma(b+n)}{\Gamma(a+n)} \int_{0}^{1} y^{a+n-1}(1-y)^{b-a-1}\left\{\int_{0}^{1}(1-x)^{n-1} e^{-n x y} d x\right\} d y
$$

The inner integral may be expressed in terms of Buckholtz's remainder function $\phi_{n}(z)$ defined in (1.5), since from (2.4) and an obvious extension of (2.3),

$$
\begin{aligned}
n \int_{0}^{1}(1-x)^{n-1} e^{-n x y} d x & ={ }_{1} F_{1}(1 ; n+1 ;-n y) \quad n=1,2, \ldots . \\
& =\phi_{n}(-y) .
\end{aligned}
$$

It then follows from (1.6) that

$$
n \int_{0}^{1}(1-x)^{n-1} e^{-n x y} d x \sim \sum_{m=0}^{\infty} c_{m}(-y) n^{-m}, \quad n \rightarrow \infty
$$

uniformly in $y$ for $0 \leqq y \leqq 1$ where, from (1.7) and (1.8), the coefficients $c_{m}(-y)$ are given by

$$
c_{0}=\frac{1}{1+y}, \quad c_{1}=\frac{y}{(1+y)^{3}}, \quad c_{2}=\frac{2 y^{2}-y}{(1+y)^{5}}, \quad c_{3}=\frac{6 y^{3}-8 y^{2}+y}{(1+y)^{7}}, \ldots
$$

Substituting the expansion (2.10) into (2.9), and employing the fact that integration of an asymptotic expansion with respect to a parameter is permissible over its domain of uniform validity $(5$, p. 16$)$, we then find

$$
\psi_{n} \sim \frac{\Gamma(b+n)}{\Gamma(b-a) \Gamma(a+n)} \sum_{m=0}^{\infty} n^{-m} \int_{0}^{1} y^{a+n-1}(1-y)^{b-a-1} c_{m}(-y) \dot{d} y .
$$

The integrals appearing in (2.12) may be evaluated by making use of Pochhammer's representation for the Gauss hypergeometric function (10, p. 20) of argument $\frac{1}{2}$

$$
\frac{\Gamma(\alpha+\beta)}{\Gamma(\alpha) \Gamma(\beta)} \int_{0}^{1} t^{\alpha-1}(1-t)^{\beta-1}(1+t)^{-\gamma} d t=2^{-\gamma} F_{1}\left(\gamma, \beta ; \alpha+\beta ; \frac{1}{2}\right)
$$


for $\operatorname{Re}(\alpha)>0, \operatorname{Re}(\beta)>0$ and arbitrary values of $\gamma$. Using (2.13), together with the expressions for the coefficients $c_{m}(-y)$ in (2.11), an expansion for $\psi_{n}$ is then obtained involving the functions ${ }_{2} F_{1}\left(\frac{1}{2}\right)$, in which $n$ appears only as a denominatorial parameter. Upon straightforward expansion of the ${ }_{2} F_{1}\left(\frac{1}{2}\right)$ functions for large $n(1, p .565)$, we finally find, after a little algebraic manipulation, the expansion

$$
\begin{aligned}
\psi_{n}=\frac{1}{2}+\frac{1}{8 n}[1+2(b-a)]+ & \frac{1}{32 n^{2}}\left[1+4(b-a)\left(\frac{3}{2}-a-b\right)\right] \\
& -\frac{1}{128 n^{3}}\left[1+8(b-a)\left\{\frac{1}{4}+a+4 b-(a+b)^{2}\right\}\right]+O\left(n^{-4}\right)
\end{aligned}
$$

as $n \rightarrow \infty$.

The expansion (2.14) has been obtained under the restriction $b>a$ and $a>-n$; as $n$ may be taken arbitrarily large, these conditions simply become $b-a>0$ for fixed values of the parameters $a$ and $b$. This restriction is a consequence of the use of the Euler integral representation for the hypergeometric function in the integral (2.5). The validity of the expansion (2.14) may, however, be extended to the domain $b-a \leqq 0$ by use of the Pochhammer loop integral (10, p. 40)

$$
{ }_{1} F_{1}(a+n ; b+n ;-n x)=\frac{\Gamma(1+a-b) \Gamma(b+n)}{\Gamma(a+n)} \cdot \frac{1}{2 \pi i} \int_{0}^{(1+)} t^{a+n-1}(t-1)^{b-a-1} e^{-n x t} d t,
$$

which holds under the conditions that $a>-n$ and $b-a$ is not a positive integer. Since (2.14) is already known to hold for $b-a>0$, this representation may be employed to cover the case $b-a \leqq 0$. Proceeding in exactly the same manner as in (2.9) and (2.10), and using the Pochhammer loop integral analogue of (2.13) (10, p. 22, Eq. (1.6.16)), yields the same expansion (2.14). It is then seen that (2.14) holds for all values of $a$ and $b$ (we exclude the values $a, b=0,-1,-2, \ldots$ as mentioned at the beginning of this section), and in particular, when $a=b,(2.14)$ reduces to Copson's expansion (1.4) for $e^{-n}$.

We now show, for $b>a>0$, that $\psi_{n}$ in (2.2) decreases monotonically from $\psi_{0}=1$ to the limit $\frac{1}{2}$ as $n$ increases from 0 to $\infty$. First consider $n$ as a continuous variable, which we denote by $x$. From (2.5) we then have for $x>0$

$$
\psi_{x}=x \int_{b}^{1}(1-t)^{x-1} F_{1}(a+x ; b+x ;-x t) d t .
$$

Denoting the hypergeometric function ${ }_{1} F_{1}(a+x ; b+x ;-x t)$ by $F(x, t)$, and differentiating $\psi_{x}$ with respect to $x$, we easily see, after an integration by parts, that

$$
\frac{\partial \psi_{x}}{\partial x}=\int_{0}^{1}(1-t)^{x}\left\{\log (1-t) \frac{\partial F}{\partial t}+\frac{x}{1-t} \frac{\partial F}{\partial x}\right\} d t .
$$

Applying Kummer's transformation $(1$, p. 505$)$ to $F(x, t)$ yields

$$
F(x, t)=e^{-x t} F_{1}(b-a ; b+x ; x t) \text {. }
$$

If the uniformly and absolutely convergent series of the type (1.9) defining the hypergeometric function on the right hand side is now differentiated term-by-term, we 
obtain the identity

$$
\frac{\partial F}{\partial x}=\frac{t}{x} \frac{\partial F}{\partial t}-e^{-x t} H(x, t)
$$

with

$$
H(x, t)=\sum_{m=1}^{\infty} \frac{(b-a)_{m}}{(b+x)_{m}} \frac{(x t)^{m}}{m !}\left[\frac{1}{b+x}+\frac{1}{b+x+1}+\ldots+\frac{1}{b+x+m-1}\right]
$$

It then follows that

$$
\frac{\partial \psi_{x}}{\partial x}=-\int_{0}^{1}(1-t)^{x}\left\{\frac{x e^{-x t}}{1-t} H(x, t)-\left[\log (1-t)+\frac{t}{1-t}\right] \frac{\partial F}{\partial t}\right\} d t \quad x>0 .
$$

Now, $H(x, t) \geqq 0$ in the range of integration for $b>0$ and $b-a>0$. By differentiation with respect to $t$ of the series defining $F(x, t)$, and application of Kummer's transformation, we find that

$$
\begin{aligned}
-\frac{\partial F}{\partial t} & =x \frac{(a+x)}{(b+x)}{ }_{1} F_{1}(a+x+1 ; b+x+1 ;-x t) \\
& =x e^{-x t} \frac{(a+x)}{(b+x)}{ }_{1} F_{1}(b-a ; b+x+1 ; x t),
\end{aligned}
$$

which is similarly positive over the range of integration for $x>0$ and $b>a>0$. Since $\log (1-t)+t /(1-t)$ is non-negative in the interval $0 \leqq t<1$, the integrand in (2.16) is non-negative and consequently $\partial \psi_{x} / \partial x<0$ for $x>0$ and $b>a>0$. It then follows, upon inserting the values $x=1,2, \ldots$, that the remainder function $\psi_{n}$ is a monotonically decreasing function of $n$ when $b>a>0$.

The extension of the domain of the parameters $a$ and $b$ for which $\psi_{n}$ is monotonic in character, is discussed further at the end of Section 3.

\section{The hypergeometric function ${ }_{1} F_{1}(a ; b ; n)$}

We now turn to the more difficult case of the hypergeometric function of positive argument ${ }_{1} F_{1}(a ; b ; n)$ which generalises Ramanujan's problem. As in Section 2, it will be supposed that neither of $a$ and $b$ is equal to zero or a negative integer. We define the remainder function $\psi_{n}$ in this case by

$$
\frac{1}{2} F_{1}(a ; b ; n)=\sum_{k=0}^{n-1} \frac{(a)_{k}}{(b)_{k}} \frac{n^{k}}{k !}+\frac{(a)_{n}}{(b)_{n}} \frac{n^{n}}{n !} \psi_{n}(n \geqq 1), \quad \psi_{0}=\frac{1}{2}
$$

so that, as in Section 2, we find

$$
\psi_{n}={ }_{2} F_{2}(1, a+n ; b+n, n+1 ; n)-\frac{1}{2} \frac{(b)_{n}}{(a)_{n}} \frac{n !}{n^{n}}{ }_{1} F_{1}(a ; b ; n) .
$$

We observe that Ramanujan's function $\theta_{n}$ for $e^{n}$ defined in (1.1) may be obtained by 
putting $a=b$ in (3.1) to find

$$
\begin{aligned}
\theta_{n} & ={ }_{1} F_{1}(1 ; n+1 ; n)-\frac{1}{2} \frac{e^{n} n !}{n^{n}} \\
& =1+\frac{1}{2} \frac{e^{n} n !}{n^{n}}\left\{1-2 \frac{\Gamma(n+1, n)}{\Gamma(n+1)}\right\} .
\end{aligned}
$$

The expansion (1.2) then follows directly by making use of the well-known expansion for large $n$ of the incomplete gamma function $\Gamma(n+1, n)(1$, p. $263 ; 7$, p. 115).

To show that $\psi_{n} \leqq \frac{1}{2}$ for $n=0,1,2, \ldots$ and $b>a>0$, we proceed by reasoning identical to that employed in (2.8) to find

$$
\begin{aligned}
{ }_{2} F_{2}(1, a+n ; b+n, n+1 ; n)= & \frac{1}{2}+\frac{1}{2} \frac{n}{\Gamma(b-a)} \frac{\Gamma(b+n)}{\Gamma(a+n)} \\
& \times \int_{0}^{1} \int_{0}^{1}(1-x)^{n-1} y^{a+n-1}(1-y)^{b-a-1}[1+(1-x) y] e^{n x y} d x d y .
\end{aligned}
$$

Employing (2.4) to express ${ }_{1} F_{1}(a ; b ; n)$ in terms of an Euler integral, we may then write $\psi_{n}$, for $b>a>0$, in the form

$$
\begin{aligned}
\psi_{n}= & \frac{1}{2}-\frac{1}{2} \frac{n \Gamma(b+n)}{\Gamma(b-a) \Gamma(a+n)} \int_{0}^{1} y^{a-1}(1-y)^{b-a-1} e^{n y}\left\{\frac{n !}{n^{n+1}}-y^{n}\right. \\
& \left.\times \int_{0}^{1}(1-x)^{n-1}[1+(1-x) y] e^{n y(x-1)} d x\right\} d y \\
= & \frac{1}{2}-\frac{1}{2} \frac{n \Gamma(b+n)}{\Gamma(b-a) \Gamma(a+n)} \int_{0}^{1} y^{a-1}(1-y)^{b-a-1} e^{n y}\left\{\frac{n !}{n^{n+1}}-\int_{0}^{y} t^{n-1}(1+t) e^{-n t} d t\right\} d y,
\end{aligned}
$$

when $n=1,2, \ldots$ It is then clearly sufficient to prove that the expression in braces is positive for $0 \leqq y \leqq 1$ and $n=1,2, \ldots$, so that the integrand is non-negative in the domain of integration.

Now, for $0 \leqq y \leqq 1$, we have

$$
\begin{aligned}
\frac{n !}{n^{n+1}}-\int_{0}^{y} t^{n-1}(1+t) e^{-n t} d t & \geqq \frac{n !}{n^{n+1}}-\int_{0}^{1} t^{n-1}(1+t) e^{-n t} d t \\
& =\frac{n !}{n^{n+1}}\left[\frac{2 \Gamma(n+1, n)}{\Gamma(n+1)}-\frac{e^{-n} n^{n}}{n !}-1\right] \\
& =\frac{e^{-n}}{n}\left(1-2 \theta_{n}\right)>0 \quad n=1,2, \ldots
\end{aligned}
$$

upon making use of (3.2) and the fact that $\frac{1}{3}<\theta_{n}<\frac{1}{2}$ for all positive integer $n$ (cf. Section 1). The result $\psi_{n} \leqq \frac{1}{2}$ for $n=0,1,2, \ldots$ and $b>a>0$ then immediately follows.

From (2.4) we have for $n=1,2, \ldots$

$$
\begin{aligned}
{ }_{2} F_{2}(1, a+n ; b+n, n+1 ; n) & =n \int_{0}^{1}(1-x)^{n-1}{ }_{1} F_{1}(a+n ; b+n ; n x) d x \\
& =n \int_{0}^{1}(1-x)^{n-1} e^{n x}{ }_{1} F_{1}(b-a ; b+n ;-n x) d x,
\end{aligned}
$$


by Kummer's transformation. The method adopted in Section 2 to determine the nature of the integral (3.3) for $n \rightarrow \infty$ by expressing it as a double integral of type (2.9) cannot readily be employed in the present case. This is because the inner integral would now involve Buckholtz's function $\phi_{n}(y)$ for $0 \leqq y \leqq 1$, instead of $\phi_{n}(-y)$, for which the expansion (1.6) is no longer uniformly valid in the neighbourhood of $y=1$. Accordingly, we proceed to obtain an asymptotic expansion, uniformly valid in $x$ in the interval $0 \leqq x \leqq 1$, of the hypergeometric function appearing in the integrand of (3.4), which may then be employed to evaluate the integral (3.4) for large values of $n$.

From (2.4) we have

$$
\begin{array}{r}
{ }_{1} F_{1}(b-a ; b+n ;-n x)=\frac{\Gamma(b+n)}{\Gamma(b-a) \Gamma(a+n)} \int_{0}^{1} t^{b-a-1}(1-t)^{a+1} e^{-n p(t)} d t, \\
\quad p(t)=x t-\log (1-t)
\end{array}
$$

for $b>a$ and $a>-n$. For $n \rightarrow \infty$, the expansion of this integral may readily be obtained by Laplace's method (8, p. 86, Theorem (8.1)) to find for $b>a$

$$
{ }_{1} F_{1}(b-a ; b+n ;-n x) \sim n^{a-b} \frac{\Gamma(b+n)}{\Gamma(a+n)} \sum_{m=0}^{\infty} \frac{(b-a)_{m}}{(1+x)^{m+b-a}} n^{-m} C_{m}(x),
$$

uniformly for $x$ in the interval $0 \leqq x \leqq 1$, where

$$
C_{0}(x)=1, \quad C_{1}(x)=1-a+\frac{1}{2} \frac{(a-b-1)}{1+x}, \ldots
$$

The restriction $b>a$ may be removed by the device described in Olver (8, p. 119, $\S 5.2)$ where, for arbitrary positive integer $M$, we define the remainder function $\phi_{M}(t)$ by

$$
t^{b-a-1}(1-t)^{a-1}=t^{b-a-1} \sum_{m=0}^{M-1}(1-a)_{m} \frac{t^{m}}{m !}+\phi_{M}(t)
$$

with

$$
\phi_{M}(t)=\sum_{m=M}^{\infty}(1-a)_{m} \frac{t^{m+b-a-1}}{m !}(|t|<1) .
$$

Then from (3.5) and (3.6) we find

$$
\begin{aligned}
& \frac{\Gamma(a+n)}{\Gamma(b+n)}{ }_{1} F_{1}(b-a ; b+n ;-n x)=n^{a-b} \sum_{m=0}^{M-1} \frac{(b-a)_{m}}{(1+x)^{m+b-a}} n^{-m} C_{m}(x) \\
& +\frac{1}{\Gamma(b-a)} \int_{0}^{1} e^{-n p(t)} \phi_{M}(t) d t .
\end{aligned}
$$

Now both sides of this equality are analytic functions of $a(>-n)$ and $b$ for $b-a+M>$ 0 , since $\phi_{M}(t)=O\left(t^{b-a+M-1}\right)$ as $t \rightarrow 0$ and the integral consequently converges for $b-a+M>0$. Then by analytic continuation with respect to $b$, this equality, proved when $b-a>0$, holds for values of $a(>-n)$ and $b$ satisfying the new condition $b-a>-M$. Application of Laplace's method to the integral involving $\phi_{M}(t)$ for $n \rightarrow \infty$ shows, since the integer $M$ is arbitrary, that (3.6) holds without restriction on $a$ and $b$. 
The uniformly valid expansion (3.6) may now be substituted in (3.4) to yield ${ }_{2} F_{2}(1, a+n ; b+n, n+1 ; n) \sim n^{a-b+1} \frac{\Gamma(b+n)}{\Gamma(a+n)} \sum_{m=0}^{\infty} \frac{(b-a)_{m}}{n^{m}} \int_{0}^{1} \frac{(1-x)^{n-1}}{(1+x)^{m+b-a}} e^{n x} C_{m}(x) d x$

We are therefore led to a consideration of integrals of the type

$$
I_{\alpha}=\int_{0}^{1}(1-x)^{-1}(1+x)^{\alpha} e^{n p(x)} d x, \quad p(x)=x+\log (1-x)
$$

for $n \rightarrow \infty$ and arbitrary bounded values of $\alpha$. The function $p(x)$ has a maximum at $x=0$, and the expansion of $I_{\alpha}$ may consequently be obtained by the saddle point method $(8$, p. 125) to find

$$
\begin{array}{r}
I_{\alpha}=\left(\frac{\pi}{2 n}\right)^{1 / 2}\left\{1+\left(\frac{2}{\pi n}\right)^{1 / 2}\left(\frac{1}{3}+\alpha\right)+\frac{1}{n}\left[\frac{1}{12}+\frac{1}{2} \alpha(\alpha-1)\right]+\left(\frac{2}{\pi n}\right)^{3 / 2} \pi\left[\frac{2}{135}+\frac{1}{6} \alpha(\alpha-1)(\alpha-3)\right]\right. \\
\left.+O\left(n^{-2}\right)\right\}
\end{array}
$$

as $n \rightarrow \infty$. This result may now be employed in (3.7) to yield, after a little algebraic manipulation, the expansion

$$
\begin{aligned}
&{ }_{2} F_{2}(1, a+n ; b+n, n+1 ; n)=n^{a-b} \frac{\Gamma(b+n)}{\Gamma(a+n)}\left(\frac{\pi n}{2}\right)^{1 / 2}\left\{1+\left(\frac{2}{\pi n}\right)^{1 / 2}\left(\frac{1}{3}+a-b\right)\right.+\frac{1}{n}\left[\frac{1}{12}+(a-1)(a-b)\right] \\
&\left.+\left(\frac{2}{\pi}\right)^{1 / 2} \frac{1}{n^{3 / 2}}\left[\frac{4}{135}+\frac{1}{6}(a-b)\{(a-b)(5 a+b-6)-4 a+5\}\right]+O\left(n^{-2}\right)\right\}
\end{aligned}
$$

To determine the expansion of $\psi_{n}$ we make use of the expansion of ${ }_{1} F_{1}(a ; b ; n)$ for large values of $n(1$, p. 508),

$$
\frac{(b)_{n}}{(a)_{n}} \frac{n !}{n^{n}}{ }_{1} F_{1}(a ; b ; n)=n^{a-b} \frac{\Gamma(b+n)}{\Gamma(a+n)}\left(\frac{e^{n} n !}{n^{n}}\right)\left\{1+\frac{(a-1)(a-b)}{n}+O\left(n^{-2}\right)\right\}
$$

provided $a, b \neq 0,-1,-2, \ldots$ and Stirling's formula

$$
\frac{e^{n} n !}{n^{n}}=(2 \pi n)^{1 / 2}\left(1+\frac{1}{12 n}+\frac{1}{288 n^{2}}-\ldots\right) . \quad n \rightarrow \infty
$$

Then from (3.1) and (3.8) we finally obtain the expansion for $n \rightarrow \infty$

$$
\begin{aligned}
\psi_{n} \sim n^{a-b} \frac{\Gamma(b+n)}{\Gamma(a+n)}\left\{\frac{1}{3}+a\right. & \left.-b+\frac{1}{n}\left[\frac{4}{135}+\frac{1}{6}(a-b)\{(a-b)(5 a+b-6)-4 a+5\}\right]+\ldots\right\} \\
= & \frac{1}{3}+a-b+\frac{1}{n}\left[\frac{4}{135}+\frac{1}{3}(a-b)\left\{(a-b)^{2}-4 a+b+3\right\}\right]+\ldots,
\end{aligned}
$$

upon employing the expansion of the gamma function ratio $(8$, p. 119)

$$
n^{a-b} \frac{\Gamma(b+n)}{\Gamma(a+n)}=1-\frac{(a-b)(a+b-1)}{2 n}+O\left(n^{-2}\right), \quad n \rightarrow \infty
$$


valid without restriction on $a$ and $b$. When $a=b$ we recover the first two terms of Ramanujan's expansion (1.2).

The determination of the domain of the parameters $a$ and $b$ for which $\psi_{n}$ in (3.1) decreases monotonically from $\psi_{0}=\frac{1}{2}$ to its limit $\frac{1}{3}+a-b$ is of a more recondite nature than that for the hypergeometric function of negative argument discussed in Section 2. From the asymptotic formula (3.9), we see that when $a-b=\frac{1}{6}$, the limiting value of $\psi_{n}$ is $\frac{1}{2}$ and it is clear that $\psi_{n}$ cannot be monotonic in this case. For $a-b<\frac{1}{6}$, a necessary condition (though clearly not sufficient) for $\psi_{n}$ to be a monotonically decreasing function of $n$ is that $\psi_{n}$ approach its asymptote $\frac{1}{3}+a-b$ from above. When $a-b>\frac{1}{6}$, a necessary condition for $\psi_{n}$ to be monotonically increasing is that the asymptote $\frac{1}{3}+a-b$ be approached from below.

We are thus led to consideration of the domains in the $(a, b)$-plane in which the function

$$
S=\frac{4}{45}+(a-b)\left\{(a-b)^{2}-4 a+b+3\right\}
$$

is either positive or negative. The curves corresponding to $S=0$ are indicated by the dashed lines in Fig. 1; $S$ is positive essentially in the left half plane and negative, apart from an intervening sector, in the right half plane. The curves $S=0$ have as asymptotes

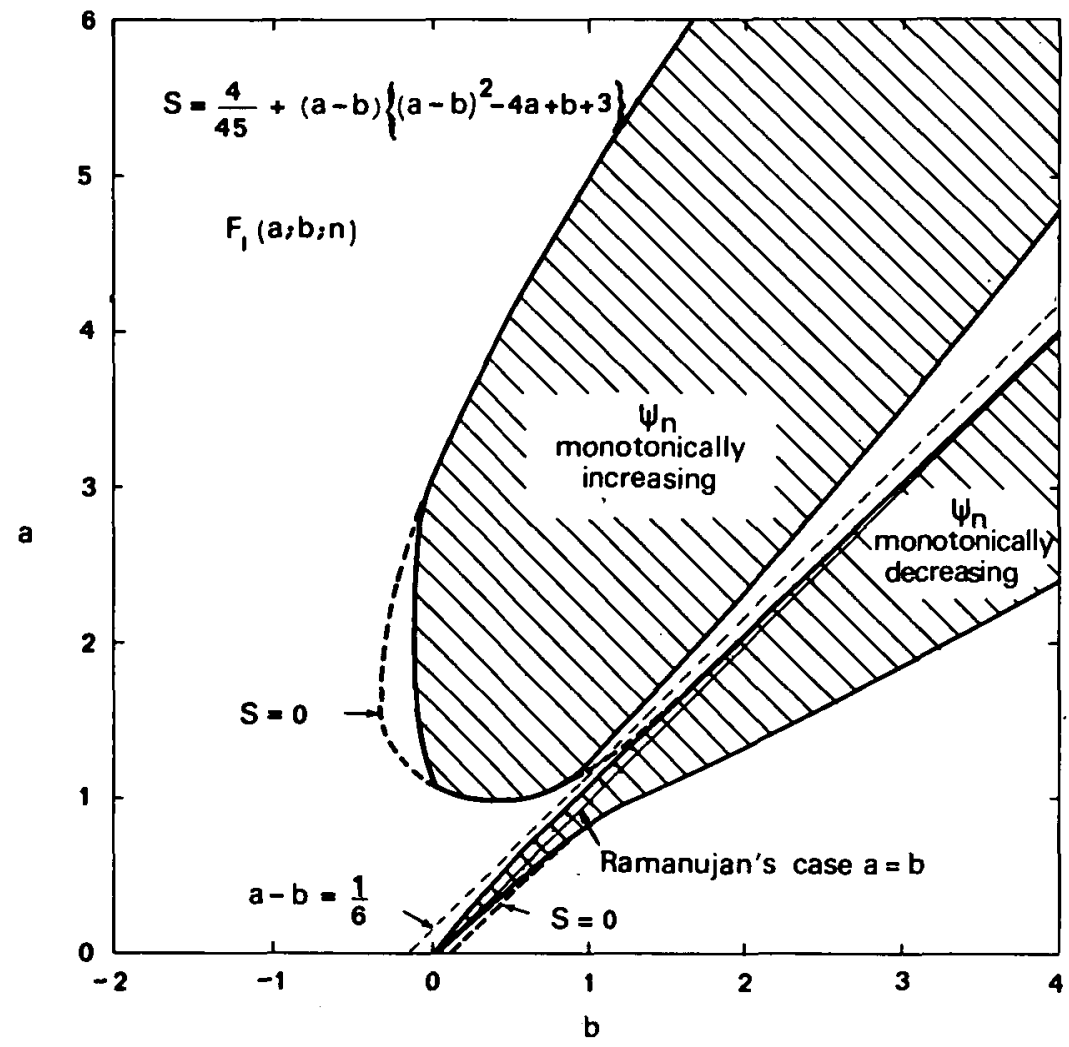

Figure 1. The domains in the $(a, b)$-plane in which the remainder function $\psi_{n}$ for ${ }_{1} F_{1}(a ; b ; n)$ is monotonic in character. 
the straight line $a=b$ and the conic $(a-b)^{2}-4 a+b+3=0$, which represents a parabola with vertex situated at $(53 / 48,-7 / 48)$ and whose axis is inclined at $\pi / 4$.

Numerical calculations of $\psi_{n}$ using (3.1) indicate that $\psi_{n}$ is monotonic in the shaded regions shown in Fig. 1. We observe that Ramanujan's case, corresponding to $a=b$, lies in the domain where $\psi_{n}$ is monotonically decreasing, in accordance with the results of Szegö and Watson mentioned in Section 1. Moreover, as $a$ and $b$ become large, the Ramanujan line $a=b$ is an asymptote of the monotonically decreasing domain. The line $a-b=\frac{1}{6}$, which separates the two monotonic domains in the $(a, b)$-plane, is also indicated. Outside of these domains, $\psi_{n}$ is found to be non-monotonic in character.

The domain in the $(a, b)$-plane for which the remainder function $\psi_{n}$ in $(2.2)$ for ${ }_{1} F_{1}(a ; b ;-n)$ is monotonically decreasing is similarly presented in Fig. 2 . In Section 2 it was shown that $\psi_{n}$ in this case is a monotonically decreasing function of $n$ in $b>a>0$, though from the asymptotic formula (2.14), it is clear that a necessary condition for monotonic behaviour is given by $a-b<\frac{1}{2}$, with $a+b \geqq 1$ when $a-b=\frac{1}{2}$. It is seen that the line $a=b$, corresponding to Copson's case for $e^{-n}$, lies within the monotonically decreasing domain and, for negative values of $a$ and $b$, would appear from numerical calculations to be the asymptote of this domain.

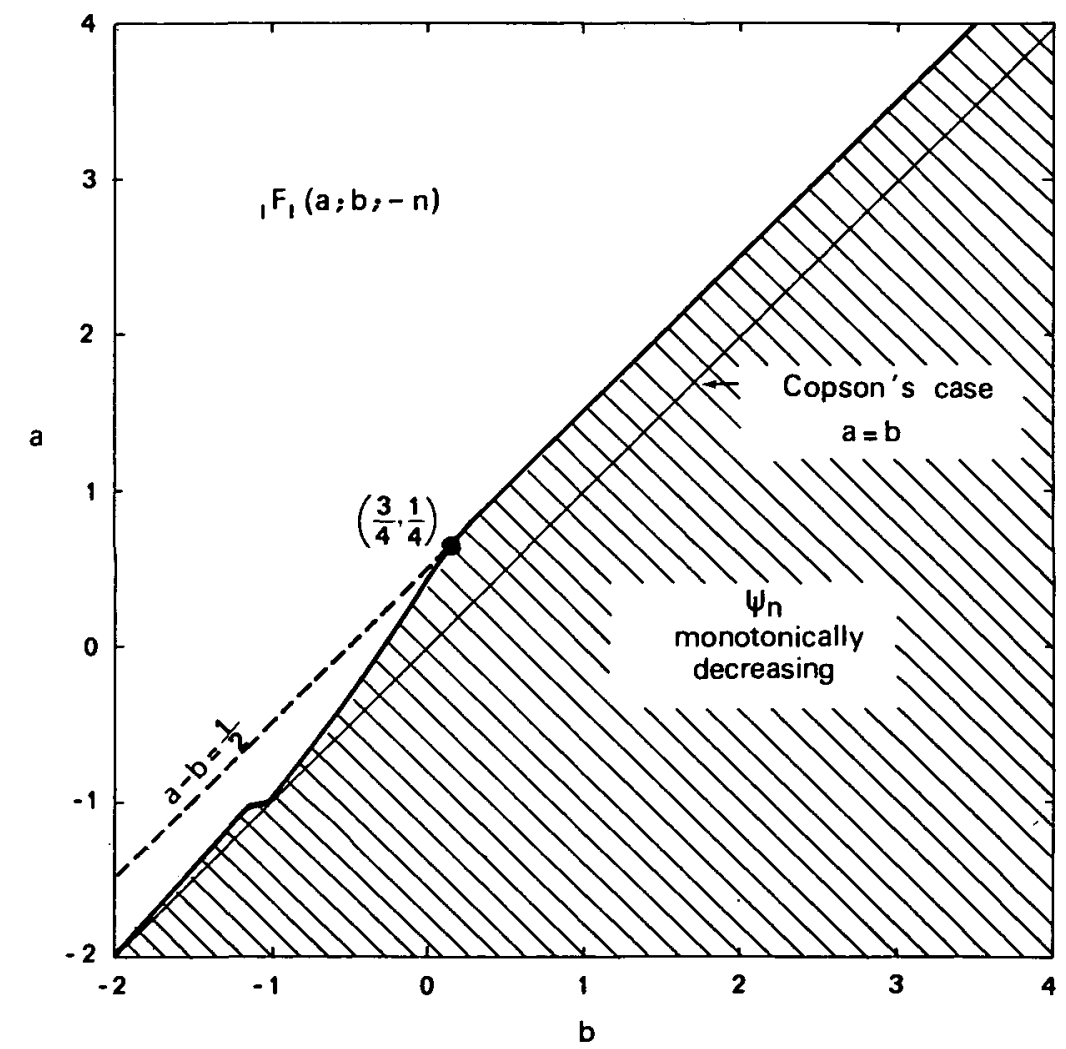

Figure 2. The domain in the $(a, b)$-plane in which the remainder function $\psi_{n}$ for ${ }_{1} F_{1}(a ; b ;-n)$ is a monotonically decreasing function of $\boldsymbol{n}$. 
4. The Bessel function $J_{0}(\lambda n), 0<\lambda \leqq 1$

In this section we discuss the Bessel function $J_{0}(\lambda n)$, for $0<\lambda \leqq 1$, and demonstrate that results analogous to those of Copson for the series $e^{-n}$ in (1.4) likewise exist for this function. We define the remainder function $\psi_{n}$ after truncation of the series for $J_{0}(\lambda n)$ at the $n$th term by

whence

$$
J_{0}(\lambda n)=\sum_{k=0}^{n-1} \frac{(-)^{k}\left(\frac{1}{2} \lambda n\right)^{2 k}}{(k !)^{2}}+(-)^{n} \frac{\left(\frac{1}{2} \lambda n\right)^{2 n}}{(n !)^{2}} \psi_{n}, \quad(n \geqq 1)
$$

$$
\psi_{n}={ }_{1} F_{2}\left(1 ; n+1, n+1 ;-\frac{1}{4} \lambda^{2} n^{2}\right), \quad \psi_{0}=1 .
$$

From (2.4) we find the integral representation

$$
\psi_{n}=n \int_{0}^{1}(1-x)^{n-1}{ }_{0} F_{1}\left(n+1 ;-\frac{1}{4} \lambda^{2} n^{2} x\right) d x
$$

for $n=1,2, \ldots$ Expressing the ${ }_{0} F_{1}$ function in terms of a Bessel function (1, p. 362), we have

$$
\begin{aligned}
\psi_{n} & =n n ! \int_{0}^{1}(1-x)^{n-1}\left(\frac{1}{2} \lambda n x^{1 / 2}\right)^{-n} J_{n}\left(\lambda n x^{1 / 2}\right) d x \\
& =1-n !\left(\frac{1}{2} \lambda n\right)^{2} \int_{0}^{1}(1-x)^{n}\left(\frac{1}{2} \lambda n x^{1 / 2}\right)^{-n-1} J_{n+1}\left(\lambda n x^{1 / 2}\right) d x
\end{aligned}
$$

after an integration by parts. For $0<\lambda \leqq 1$, the above integrands are both non-negative in the range of integration, since the smallest positive zeros $j_{n, 1}$ and $j_{n+1,1}$ of $J_{n}(z)$ and $J_{n+1}(z)$ respectively satisfy $j_{n+1,1}>j_{n, 1}>n(14, \S \S 15.22,15.3)$. It therefore follows that $0<\psi_{n}<1$ for $n=1,2, \ldots$ and $0<\lambda \leqq 1$.

To determine the expansion of $\psi_{n}$ for large values of $n$, we first observe that the function $y=\left(\frac{1}{2} \lambda n x^{1 / 2}\right)^{-n} J_{n}\left(\lambda n x^{1 / 2}\right)$, appearing in the integrand in (4.2), is a monotonically decreasing function of $x$ for $0 \leqq x \leqq 1$ and for fixed $\lambda$ in $0<\lambda \leqq 1$. This follows from the fact that its derivative

$$
\frac{d y}{d x}=-\frac{1}{x}\left(\frac{1}{2} \lambda n x^{1 / 2}\right)^{-n+1} J_{n+1}\left(\lambda n x^{1 / 2}\right)<0 \quad(0<\lambda \leqq 1)
$$

for $x$ in the interval $0 \leqq x \leqq 1$, since $j_{n+1,1}>n$. Accordingly, we may define the new variable $\tau(x)$ by

$$
e^{-\tau}=(1-x)\left[n !\left(\frac{1}{2} \lambda n x^{1 / 2}\right)^{-n} J_{n}\left(\lambda n x^{1 / 2}\right)\right]^{1 / n},
$$

where $\tau$ increases monotonically from 0 to $\infty$ as $x$ describes the path of integration in (4.2) from 0 to 1 , and write (4.2) in the form

$$
\psi_{n}=n \int_{0}^{\infty} e^{-n \tau} q(n, \tau) d \tau
$$

where, by differentiating (4.3),

$$
q(n, \tau)=\frac{1}{1-x} \frac{d x}{d \tau}=\left\{1+\frac{1}{2} \lambda \frac{(1-x)}{x^{1 / 2}} \frac{J_{n+1}\left(\lambda n x^{1 / 2}\right)}{J_{n}\left(\lambda n x^{1 / 2}\right)}\right\}^{-1} .
$$


From (4.3) we find the expansion

$$
\tau=\alpha x+\frac{1}{2} \beta x^{2}+\frac{1}{3} \gamma x^{3}+\ldots
$$

with

$$
\alpha=1+\frac{\lambda^{2}}{4} \frac{n}{n+1}, \quad \beta=1+\frac{\lambda^{4}}{16} \frac{n^{3}}{(n+1)^{2}(n+2)}, \quad \gamma=1+\frac{\lambda^{6}}{32} \frac{n^{5}}{(n+1)^{3}(n+2)(n+3)} .
$$

Since $\tau^{\prime}(0)$ is non-zero, there exists an expansion $x=x(\tau)$ valid in the neighbourhood of $\tau=0(6, \S 12.051)$ such that

$$
q(n, \tau)=\frac{1}{\alpha}\left\{1+\left(1-\frac{\beta}{\alpha}\right) \frac{\tau}{\alpha}+\left[1-\frac{3 \beta}{2 \alpha}+\frac{1}{\alpha^{2}}\left(\frac{3}{2} \beta^{2}-\alpha \gamma\right)\right]\left(\frac{\tau}{\alpha}\right)^{2}+\ldots\right\}
$$

valid for sufficiently small $|\tau|$.

In order to apply Watson's lemma to the determination of the integral (4.4) for $n \rightarrow \infty$, we first observe from (4.5) that on the path of integration the ratio of the Bessel functions is positive $(\lambda \leqq 1)$ and $0<q(n, \tau) \leqq 1$. Secondly, to show that the series expansion (4.6) for $q(n, \tau)$ has a finite radius of convergence, we consider the singular points of the function $d x / d \tau$. From (4.5)

$$
\frac{d \tau}{d x}=\frac{1}{1-x}+\frac{1}{2} \lambda x^{-1 / 2} \frac{J_{n+1}\left(\lambda n x^{1 / 2}\right)}{J_{n}\left(\lambda n x^{1 / 2}\right)},
$$

which is an integral (entire) function of $x$ possessing simple poles at $x=1$ and $\lambda n x^{1 / 2}=j_{n, k}, k=1,2, \ldots$, where $j_{n, k}$ denotes the $k$ th positive zero of $J_{n}(z)$. These values of $x$ correspond to infinite values of $\tau$. By considering the graph of $d \tau / d x$ as a function of $x$ (real), it is readily seen that there is an infinite sequence of positive values of $x$, situated between successive zeros of $J_{n}\left(\lambda n x^{1 / 2}\right)$ and $J_{n+1}\left(\lambda n x^{1 / 2}\right)$, at which $d \tau / d x$ vanishes. To verify that there are no complex zeros of $d \tau / d x$ we consider the change of argument of $f\left(x^{1 / 2}\right) \equiv d \tau / d x$ round a rectangular contour described in the positive sense in the complex $x^{1 / 2}$-plane with vertices at $\pm i T, \pm i T+\left(k+\frac{1}{2} n+\frac{1}{4}\right) \pi, T>0$ (so that the side parallel to the imaginary axis separates the $k$ th and $(k+1)$ th zeros of $\left.J_{n}\left(\lambda n x^{1 / 2}\right)\right)$. Then in the manner described in $(14, \S 15.4)$ the change of argument of $f$ round the contour may be shown to be $-2 \pi$ as $T \rightarrow \infty$ and $k$ large. It then follows that the only zeros of $d \tau / d x$ are the real positive zeros which interlace with those of $J_{n}\left(\lambda n x^{1 / 2}\right)$ and $J_{n+1}\left(\lambda n x^{1 / 2}\right)$. Since $j_{n, 1}>n$ and $0<\lambda \leqq 1$, this infinite sequence of zeros lies entirely to the right of the point $x=1$. From (4.3) the corresponding values of $\tau$ at these zeros must therefore satisfy $|\operatorname{Im}(\tau)| \geqq \pi$. We consequently infer that the inversion $x=x(\tau)$ of (4.3) is regular in at least $|\tau|<\pi$.

The conditions of Watson's lemma (14, p. 236) are therefore satisfied. Expanding the coefficients $\alpha, \beta, \gamma$ in (4.5) in terms of $1 / n$, we may now apply the extension of Watson's lemma (8, p. 326 Theorem 2.1) to the integral (4.4) when $q=q(n, \tau)$ to find the expansion

$$
\psi_{n}=\frac{4}{4+\lambda^{2}}+\frac{32 \lambda^{2}}{\left(4+\lambda^{2}\right)^{3} n}-\frac{16 \lambda^{2}}{\left(4+\lambda^{2}\right)^{5} n^{2}}\left(\lambda^{4}-32 \lambda^{2}+48\right)+O\left(n^{-3}\right)
$$

for $n \rightarrow \infty$. The expansion of $\psi_{n}$ in the particular case $\lambda=1$, corresponding to $J_{0}(n)$, is 
given by

$$
\psi_{n}=\frac{4}{5}+\frac{32}{125 n}-\frac{272}{3125 n^{2}}-\frac{2816}{15625 n^{3}}+O\left(n^{-4}\right)
$$

It is of interest to compare the values of $\psi_{n}$ obtained from the asymptotic formula (4.8) with those calculated directly from the power series expansion of the ${ }_{1} F_{2}$ function in (4.1) with $\lambda=1$. The values of $\psi_{n}$ for several values of $n$ are presented in the following table:

\begin{tabular}{rcc}
\hline$n$ & $\begin{array}{c}\text { Exact value of } \psi_{n} \\
\text { with } \lambda=1\end{array}$ & $\begin{array}{c}\text { Asymptotic value } \\
\text { from (4.8) }\end{array}$ \\
\hline 5 & 0.846528 & 0.846277 \\
10 & 0.824561 & 0.824549 \\
15 & 0.816628 & 0.816626 \\
20 & 0.812560 & 0.812560 \\
\hline
\end{tabular}

It will be seen that surprisingly good accuracy is obtained for values of $n$ as small as $n=5$ and that for $n \geqq 20$, the expansion (4.8) yields the value of $\psi_{n}$ accurate to at least the sixth decimal figure.

We now show that $\psi_{n}$ in (4.1) decreases monotonically from the value $\psi_{0}=1$ to its limit $4 / 4\left(+\lambda^{2}\right)$ as $n$ increases from 0 to $\infty$. The proof on the lines of that used for monotonicity of the remainder function for ${ }_{1} F_{1}(a ; b ;-n)$ in Section 2 appears to be somewhat more difficult in this case. This results from the fact that in the corresponding integral representation of type (2.15) defining $\partial \psi_{x} / \partial x$ for $J_{0}(\lambda n)$, the integrand is found to change sign in the range of integration. We therefore proceed in a different manner and consider the difference

$$
\begin{aligned}
\psi_{n+1}-\psi_{n} & ={ }_{1} F_{2}\left(1 ; n+2, n+2 ;-\frac{1}{4} \lambda^{2}(n+1)^{2}\right)-{ }_{1} F_{2}\left(1 ; n+1, n+1 ;-\frac{1}{4} \lambda^{2} n^{2}\right) \\
& =\{(n+1) !\}^{2} \sum_{r=1}^{\infty}(-)^{r} u_{r} \quad n=0,1,2, \ldots
\end{aligned}
$$

where

$$
u_{r}=\frac{\left(\frac{1}{2} \lambda n\right)^{2 r} \delta_{r}}{\{(n+r+1) !\}^{2}}, \quad \delta_{r}=\left(\frac{n+1}{n}\right)^{2 r}-\left(\frac{n+r+1}{n+1}\right)^{2}
$$

It is easily seen by application of the binomial theorem that $\delta_{r}>r(r+1) /(n+1)^{2}>0$ for $r, n=1,2, \ldots$ and that the coefficients $u_{r}$ are positive for $n=0,1,2, \ldots$ Since (4.9) converges absolutely, we may regroup its terms and write

$$
\psi_{n+1}-\psi_{n}=-\{(n+1) !\}^{2} \sum_{k=0}^{\infty}\left(u_{2 k+1}-u_{2 k+2}\right),
$$

where the sign of $u_{2 k+1}-u_{2 k+2}$ depends upon the sign of the quantity

$$
\Delta_{n, k}(\lambda)=n^{4 k+2}\left\{\delta_{2 k+1}-\frac{1}{4} \frac{\lambda^{2} n^{2}}{(n+2 k+3)^{2}} \delta_{2 k+2}\right\} \quad n=0,1,2, \ldots
$$


We will now show that $\Delta_{n, k}(\lambda)>0$ for $n=1,2, \ldots, k=0,1,2, \ldots$ and $0<\lambda \leqq 1$, so that the difference between sucessive coefficients $u_{2 k+1}-u_{2 k+2}$ in (4.10) is positive. Since the case $n=0$ is trivial with $\psi_{0}=1$ and $\psi_{1}<1$, it then follows that for $n=$ $0,1,2, \ldots \psi_{n+1}-\psi_{n}<0$, and therefore that the remainder function $\psi_{n}$ in (4.1) for $J_{0}(\lambda n)$ is a monotonically decreasing function of $n$. For $n>0$, the most stringent case arises when $\lambda=1$ since $\delta_{r}>0$, and $\Delta_{n, k}(\lambda) \geqq \Delta_{n, k}(1)$, where

$$
\begin{aligned}
\Delta_{n, k}(1) & =n^{4 k+2}\left\{\left(\frac{n+1}{n}\right)^{4 k+2}\left[1-\frac{1}{4} \frac{(n+1)^{2}}{(n+2 k+3)^{2}}\right]+\frac{1}{4}\left(\frac{n}{n+1}\right)^{2}-\left(\frac{n+2 k+2}{n+1}\right)^{2}\right\} \\
& =\frac{n^{4 k+4}}{(n+1)^{2}}\left\{\left(\frac{n+1}{n}\right)^{4 k+4}\left[\frac{3}{4}+\frac{(k+1)(n+k+2)}{(n+2 k+3)^{2}}\right]+\frac{1}{4}-\left(1+\frac{2 k+2}{n}\right)^{2}\right\} \\
& >\frac{n^{4 k+4}}{(n+1)^{2}}\left\{\left(\frac{n+1}{n}\right)^{4 k+4} \frac{(k+1)(n+k+2)}{(n+2 k+3)^{2}}-\frac{k+1}{n}\right\} \\
& >\frac{n^{4 k+4}}{(n+1)^{2}} \frac{k(k+1)}{(n+2 k+3)^{2}} \quad k=0,1,2, \ldots ; n=1,2, \ldots
\end{aligned}
$$

upon repeated application of the binomial theorem. Therefore $\Delta_{n, k}(1)>0$ for $n=$ $1,2, \ldots, k=0,1,2, \ldots$ and the result follows.

As a consequence of the monotonic character of $\psi_{n}$, we remark that $4 /\left(4+\lambda^{2}\right)<\psi_{n}<$ 1 for all positive integer $n$. This result may be employed to derive rational bounds for $J_{0}(\lambda n)$ when $0<\lambda \leqq 1$. This will be discussed more fully, together with the Bessel function $J_{n}(\lambda n)$ of argument nearly equal to its order, in a future paper.

The results of this section may be easily generalised to the Bessel function $\left(\frac{1}{2} \lambda n\right)^{-\nu} J_{\nu}(\lambda n)$ with the corresponding remainder function

$$
\psi_{n}={ }_{1} F_{2}\left(1 ; n+1, n+\nu+1 ;-\frac{1}{4} \lambda^{2} n^{2}\right) .
$$

By means of similar analysis, we then find the asymptotic formula

$$
\psi_{n}=\frac{4}{4+\lambda^{2}}+\frac{4 \lambda^{2}}{\left(4+\lambda^{2}\right)^{2}}\left(\nu+\frac{8}{4+\lambda^{2}}\right) \frac{1}{n}+O\left(n^{-2}\right)
$$

for large values of $n$. It is to be noted that the limit of $\psi_{n}$ is independent of the order $\nu$.

\section{Concluding remarks}

We conclude with some remarks of a general nature concerning the higher order hypergeometric function ${ }_{p} F_{q}(-n)$, defined in (1.9) with $q \geqq p \geqq 0$, where the parameters $a_{1}, \ldots, a_{p}$ and $b_{1}, \ldots, b_{q}$ are arbitrary complex numbers. It is readily shown that $\psi_{n}$, defined in the manner (2.1), may be expressed as

$$
\psi_{n}={ }_{p+1} F_{q+1}\left(1, a_{1}+n, \ldots, a_{p}+n ; n+1, b_{1}+n, \ldots, b_{q}+n ;-n\right)
$$

provided none of the $a_{r}, r=1, \ldots, p$ and $b_{r}, r=1, \ldots, q$ equals zero or a negative integer.

When $p<q$, there is an excess $s=q-p$ of denominatorial parameters and the determination of the behaviour of $\psi_{n}$ for large values of $n$ may be easily obtained by 
formally expanding the series defining ${ }_{p+1} F_{q+1}(-n)$ to find

$$
\psi_{n}=1-n^{-s}+O\left(n^{-s-1}\right) . \quad n \rightarrow \infty
$$

Since $\psi_{0}=1$, it is evident that $\psi_{n}$ in this case cannot be monotonic in character.

The most interesting case occurs when $p=q$, when the numbers of denominatorial and numeratorial parameters are equal. It is conjectured in this case that

$$
\psi_{n}=\frac{1}{2}+\frac{1}{8 n}\left[1+2 \sum_{r=1}^{p}\left(b_{r}-a_{r}\right)\right]+O\left(n^{-2}\right), \quad n \rightarrow \infty
$$

a result which is in accordance with Copson's result when $p=q=0$ and (2.14) when $p=q=1$. It is seen that the limit of $\psi_{n}$ is always equal to $\frac{1}{2}$ for the generalised hypergeometric function ${ }_{p} F_{p}(-n), p=1,2, \ldots$

We remark, as noted by Buckholtz in the case of the exponential function, that the corresponding discussion of the function ${ }_{p} F_{q}(n)$ of positive arguement is of a more recondite nature.

Acknowledgement. The author wishes to acknowledge the assistance of Mrs $\mathrm{N}$. Auby with the numerical calculations undertaken in Figs. 1, 2 and Section 4 and the referee for some constructive criticism.

\section{REFERENCES}

(1) M. Abramowttz and I. Stegun (Eds.), Handbook of Mathematical Functions (Dover, New York; 1965).

(2) J. D. Buckнoltz, Concerning an approximation of Copson, Proc. Amer. Math. Soc. 14 (1963), 564-568.

(3) L. Carlirz, The coefficients in an asymptotic expansion, Proc. Amer. Math. Soc. 16 (1965), 248-252.

(4) E. T. Copson, An approximation connected with $e^{-x}$, Proc. Edinburgh Math. Soc. (2), 3 (1932), 201-206.

(5) A. ERdélyi, Asymptotic Expansions (Dover, New York; 1965).

(6) H. Jefrereys and B. S. Jeffreys, Methods of Mathematical Physics (Cambridge Univ. Press, 1972).

(7) D. E. KNuTH, Fundamental Algorithms (Series in Computer Science and Information Processing, Addison-Wesley, 1973).

(8) F. W. J. Olver, Asymptotics and Special Functions (Academic Press, 1974).

(9) S. Ramanujan, J. Ind. Math. Soc. 3 (1911), 128; ibid. 4 (1911), 151-152; Collected Papers (Chelsea, New York; 1962), 323-324.

(10) L. J. Slater, Generalised Hypergeometric Functions (Cambridge Univ. Press, 1966).

(11) G. G. STOKEs, Note on the determination of arbitrary constants which appear as multipliers of semi-convergent series, Proc. Camb. Phil. Soc. 6 (1889), 362-366.

(12) G. Szegö, Uber einige von S. Ramanujan gestellte Aufgaben, J. London Math. Soc. 3 (1928), 225-232. 
(13) G. N. WAtson, Theorems stated by Ramanujan: approximations connected with $e^{x}$, Proc. London Math. Soc. 29 (1928), 293-308.

(14) G. N. Watson, Theory of Bessel Functions (Cambridge Univ. Press, 1944).

(15) R. Wong, On uniform asymptotic expansion of definite integrals, J. Approx. Theory 7 (1973), 76-86.

\begin{abstract}
Association Euratom-CEA
Centre d'Etudes Nucleaires

92260 FONTENAY-AUX-ROSES

FrANCE
\end{abstract}

